\title{
Instrument Development and Validation of the Comprehensive Ability of Older People Assessment scale
}

\author{
Weitong Li \\ Yuan Zhu \\ Nanjing University of Chinese Medicine \\ Yujing Chen \\ Nanjing University of Chinese Medicine \\ Yalou Pu \\ Suzhou Vocational Health College \\ Guihua Xu (D357496717@qq.com) \\ Nanjing University of Chinese Medicine
}

Nanjing University of Chinese Medicine https://orcid.org/0000-0003-3267-3085

\section{Research article}

Keywords: Comprehensive Ability, older People, instrument development, nursing, reliability, validity

Posted Date: November 10th, 2020

DOI: https://doi.org/10.21203/rs.3.rs-103115/v1

License: (c) (1) This work is licensed under a Creative Commons Attribution 4.0 International License. Read Full License

Version of Record: A version of this preprint was published at Nursing Open on August 6th, 2021. See the published version at https://doi.org/10.1002/nop2.1020. 


\section{Abstract}

Background: At present, many aging assessment instruments have been developed at home and abroad, but the quality of care service in China's pension institutions is uneven, and there is still a lack of a unified tool for assessing the ability of the elderly uesd by evaluators of long-term care institutions.

Method: The Comprehensive Ability of Older People Assessment scale was completed by a sample of 971 older people from 4 long-term care facilities in China and the data were collected between April 2018-December 2019. One-way analysis of variance and multiple regression analysis were used to screen scale items, while focus group interviews were used to integrate the subjective and objective items. Confirmative factor analysis and expert judgment were applied to explore construct validity. Reliability was explored through internal consistency estimation using Cronbach's alpha and homogeneity evaluation using corrected item-total correlations. Cluster analysis and discriminant analysis were used to segment the comprehensive ability assessment scores and discriminant function was established to determine the boundary value of each segment, with correlation analysis used to perform reverse verification.

Results: Factor analysis yielded 40 items with six dimensions, including 'Mentation and cognitive,' 'Perception and communication,' 'Emotional problems,' 'Mental and behavioral problems,' 'Daily life and social participation,' and 'Skin and oral status'. The Cronbach's a was 0.951 while the dimensions show a Cronbach's a 0.760-0.946.

The rationality and scientificity of the scale was proved by the correlation analysis of reverse validation.

Conclusion: The 4-grade Comprehensive Ability of Older People Assessment Scale had good validity and reliability and should be considered for institutional assessors.

\section{1 | Background}

The trend of population aging began in Europe in the middle of the 19th century. Since the 1970s, it has gradually spread to countries in North America and Asia. The proportion of older people and over-aged population and the dependency ratio of the elderly have always been rising, which has become a global issue affecting human development[1]. Population aging is a universal, natural, sustained and inevitable phenomenon, which has an impact on the elderly themselves and the country where they live in. The phenomenon of aging not only brings physiological changes to older people, but also leads to emotional, cognitive and social changes[2]. If there is no way to actively deal with aging and accurately assess and pay attention to the health status of older people, the contradiction of aging will become more prominent and there will be more adverse social, economic and political consequences in the near future[3].

The active aging policy is based on meeting the needs of the elderly and emphasizing that they maintain good physical, psychological and intellectual conditions, so as to prevent or delay the adverse physiological, psychological or social changes of the elderly, eliminate the marginalization and morbidization of the elderly group, and transform the elderly from concentrators of social problems to solvers, creators and driving forces to promote sustainable development[4]. Aging assessment instruments can objectively and multidimensionally evaluate the risks and adverse outcomes of the elderly, so as to provide a basis for doctors and nurses to better formulate care plans and allocate resources. at the same time, it can also ensure that the elderly in need can be intervened as soon as possible, delay the process of disability and improve the quality of care in pension institutions[5]. At present, many aging assessment instruments have been developed at home and abroad, but the quality of care service in China's pension institutions is uneven, and there is still a lack of a unified tool for assessing the ability of the elderly.

For these reasons, to evaluate the physiological, social, functional and other dimensions of older people effectively comprehensively is the fundamental solution and premise for dealing with the future crisis and aging of disabled elderly. Compared with traditional medical assessments, it has been found that comprehensive geriatric assessment (CGA) can improve the elderly's activity of daily living and cognitive function, improve quality of life, reduce medical demands and expense, and save health resources at the same time[6]. At present, there are many assessment tools for the elderly recognized both domestically and internationally, but some scales are too miscellaneous and the assessment time is too long to be implemented on a large scale. In addition, the quality of nursing care services in long-term care facilities, communities and hospitals is uneven, so there is 
still a lack of a unified comprehensive assessment tool for older people. Therefore, the establishment of a comprehensive and effective Comprehensive Ability of Older People Assessment Scale can objectively and multi-dimensionally assess the risks and adverse outcomes of older people, so as to provide the basis for medical staff to better formulate care programs and allocate resources.

After a systematic review of recent developments in comprehensive geriatric assessment instruments in long-term care facilities, our research team found that the International Resident Assessment Instrument-Long-term care facillities (InterRAI-LTCF) and the Ability assessment questionnaire for older people (abbreviation: AAQ) have formed a mature system, and they are comprehensive assessment tools for the elderly which are worth popularizing in the long-term care institutions of our country in the future. Our research team used these two scales in the actual investigation of long-term care facilities and found some problems. InterRAlLTCF was originated from the minimum data set (MDS) developed by the American government in 1987 to unify the evaluation standards of care institutions and nursing homes[7]. As a scale consisting of 196 items in 14 dimensions, the assessment contents of InterRAI-LTCF mainly include identity information, cognitive ability, communication and vision, emotion and behavior, social and psychological health, physical function, self-control of urination and defecation, disease diagnosis, health status, oral and nutritional status, skin condition, recreational activities and social participation. InterRAI-LTCF assessment data are mainly obtained through observation, which is less invasive than other types of scales, and it has been used as a standardized comprehensive assessment tool for assessing disability status of the elderly in more than 40 countries and regions, which is proved to have good reliability and validity[8]. However, some of the items in InterRAI-LTCF are not closely related to the nursing care of the elderly. At present, the research on the application of InterRAI-LTCF in long-term care institutions is insufficient. Boorsma[9] tried to implement InterRAI-LTCF in 10 nursing homes in Netherlands, then they found that the management methods and financial support of residential care homes needed to undergo major changes and sufficient personnel and computer equipment were needed to support the implementation of InterRAI-LTCF at the same time. In the investigation phase, it was also found that InterRAI-LTCF was time-consuming and had too many items. Due to the lack of resources in long-term care institutions, it is difficult to implement this scale.

The other available instrument is AAQ[10], implemented by the Chinese Ministry of Civil Affairs in 2013 to evaluate the older people's grade of ability. This is a 40-item scale with a 4-grade assessment of 4 dimensions, including the elderly's Activities of daily living $(A D L)$, spiritual cognition, perception and communication, and social participation. According to results of this questionnaire evaluation, older people can be divided into 4 grades: intact ability, mild disability, moderate disability and severe disability. The Cronbach's a coefficient of the questionnaire is $0.725[11]$. It was found that the focus of this scale is ability classification which does not include the condition of the elderly, while the questionnaire items are too simple to affect the accuracy of the assessment. In conclusion, the two available instruments at present do not have universality and reliability. Also, they are not specific for comprehensive assessment of older people's ability.

\section{2 | Methods}

\section{1| Aim}

The aim of this study was to develop a new instrument, the Comprehensive Ability of Older People Assessment scale (CAOPAs), which should be built on combining items from the two current scales (InterRAI-LTCF and AAQ), bearing the purpose to test its psychometric characteristics and to classify the ability grades of CAOPAs.

\section{2 | Methodology}

The cross-sectional design was used. In this study, the validity and reliability of CAOPAs were considered, and the scales were classified.

\section{3 | The validity}

The content validity was assessed by the expert judgment method, including content validity of the item (Item-level CVI, I-CVI) and content validity index of scale grade (Scale-level CVI, S-CVI) [12]. The evaluation method sets the option to four grades: $1=$ irrelevant; 2 = weak correlation; 3 = strong correlation; 4 = very relevant. Count the number of experts with "3 points = strong correlation" and "4 points = very relevant" for each item, and divide the number of experts counted by the total number of experts 
participating in the evaluation to get the corresponding $\mathrm{I}-\mathrm{CVI}$. When the number of experts is more than $6, \mathrm{I}-\mathrm{CVI}$ is required to be no less than 78. S-CVI/UA is the percentage of all items whose scores are 3 or 4 , which is more than $80 \%$, indicating that the content validity of the scale is good. S-CVI/Ave is expressed by the I-CVI mean of all items of the scale, and when the S-CVI/Ave value of the scale is $>0.9$, indicating that it meets the requirements. At the beginning of this study, the theoretical framework has been established according to the purpose of it, the scale selected in the early stage and the results of focus interviews. It is generally believed that exploratory factor analysis is not necessary in the development of the scale on the basis of previous studies and conclusions[13]. The construct validity of CAOPAs was assessed through confirmative factor analysis (CFA). SPSS 22.0 software (SPSS, Inc.) was used for all statistical analysis except for CFA, where the Mplus 7.1 software was used. Spearman correlation analysis was adopted to perform reverse verification of the scale.

\subsubsection{Confirmative factor analysis}

The standard of good fit varies, namely the model fit for the CFA considered $\chi 2$, the comparative fit index (CFI) (values $>0.95$ indicated a good fit), the root mean square error of approximation (RMSEA) (values $<0.06$ indicated a good fit), the normed fit index or the Tucker Lewis index (TLI) (values $\geq 0.95$ indicated a good fit), and the standardized root mean square residual (SRMR) (values $<0.08$ indicated a good fit).

\subsection{2 | Reverse verification}

For reverse verification, whether the results assessed by CAOPAs are more convincing was verified. This study used CAOPAs and AAQ to evaluate the same group of elderly people, and spearman correlation analysis was conducted between the grades $(1,2,3,4)$ of older people with ability grade difference and cognitive score, activities of daily living, depression score, pain score, communication score and pressure sore score in InterRAI-LTCF, whose results showed that the correlation coefficient of CAOPAs was higher than that of $A A Q$, which meant that CAOPAs was more related to the ability and health status of the elderly. Thus the results and scale classification were more convincing.

\section{4 | The reliability}

CAOPAs was tested for internal consistency with Cronbach's a. For internal consistency, a coefficient a of 0.70 or greater was used as standard[14]. For external consistency, which was measured by half-reliability, the judgment standard can be compared with the measurement standard of a reliability coefficient.

\section{5 | Scale classification}

For the classification of CAOPAs, K-means algorithm and Fisher linear discriminant analysis were used to classify the samples. The clustering results were evaluated by four validity indexes, within-cluster sum of squared errors (SSE), single side band (SSB), Intra-Cluster part of Dispersion (IntraDPS(K)) and Inter-Cluster part of Dispersion (InterDPS(K)), so as to establish the best K value and update the values of each cluster center step by step and get the best clustering results according to the evaluation results[15].

\subsection{Participants}

From April 2018 to January 2019, the research team selected 725 elderly people from three long-term care facilities in China in accordance with convenience sampling method for investigation. After the scale was formed and the reliability and validity were tested, 246 elderly people were selected from the fourth pension institution for reverse validation and grading from October to December 2019. Inclusion criteria included: 『signed written informed consent; $₫$ Age $\geq 60$ years old; $₫$ stay in the long-term care facilities for more than 3 months; 『volunteer for the research group study; 『The elderly or their caregivers, such as nursing staff and nurses, have the ability to accept the questions on the questionnaire from the assessors and answer the relevant questions correctly. Exclusion criteria included: 邓those who fill in the form incorrectly or incompletely; 邓those who are unable to continue the investigation due to sudden emergency during the research process; $\triangle$ the elderly who leave the institution and live outside during the investigation.

\section{7 | Instrument}

Page $4 / 18$ 
In the early stage, the research team established the theoretical framework of CAOPAs according to the research purpose, the scale selected by literature reading and field investigation (InterRAI-LTCF and AAQ) and the results of focus interview.

\subsection{Ethical considerations}

This study was approved by Nanjing University of Chinese Medicine Ethics Committee. Before data collection, signed informed consent were obtained from all participants. Based on the ethical principles of informed consent, respect, beneficence and confidentiality, as honoured by the research team, all participants were informed about the aims and the method of the study. In the case of the elderly with cognitive decline, the legal guardian or representative to provide consent to participate in our study. Benefits and possible risks associated with this study were also explained to the participants. Besides, all data were collected anonymously and archived in a password-protected computer. Only the primary investigator and research team members have access to the data.

\section{3 | Results}

\section{1 | Participant characteristics}

In this study, a total of 775 older people from three long-term care facilities in China were included. 725 questionnaires were collected for the first time, of which 25 were discharged, 3 died and 22 were transferred to hospital for treatment, thus the effective recovery rate was $93.5 \%$. The average age of sample was 84.6 (SD 9.2) (Table 1). The statistics of chronic diseases are hypertension (53.7\%), diabetes mellitus (23.4\%), coronary heart disease (17.9\%) and cerebrovascular disease (35.2\%). Mild disability and severe disability account for a relatively high proportion in the ability grade of the elderly, with the percentages of $40.8 \%$ and $40.7 \%$ (Non-zero p-values < 0.001) (Table 2). 
Table 1

Older people's socio-demographic characteristics

\begin{tabular}{|ll|}
\hline Characteristics & Older people $(\mathbf{n}=\mathbf{7 2 5})(\%)$ \\
\hline Age(years old), $\mathrm{n}(\%)$ & \\
\hline $60 \sim 69$ & $39(5.4)$ \\
\hline $70 \sim 79$ & $160(22.0)$ \\
\hline$\geq 80$ & $526(72.6)$ \\
\hline Gender, $\mathrm{n}(\%)$ & \\
\hline Male & $279(38.5)$ \\
\hline Female & $446(61.5)$ \\
\hline Marital status, $\mathrm{n}(\%)$ & \\
\hline Unmarried & $26(3.6)$ \\
\hline Married & $326(45.0)$ \\
\hline Widowed & $352(48.6)$ \\
\hline Divorced & $2(0.3)$ \\
\hline No statement of marital status & $19(2.6)$ \\
\hline Degree of education, $\mathrm{n}(\%)$ & \\
\hline Junior high school and below & $281(38.8)$ \\
\hline High school / Vocational high school & $146(20.1)$ \\
\hline University and above & $175(24.1)$ \\
\hline Unknown & $123(17.0)$ \\
\hline Medical expense & \\
\hline Part of the expenses & $3(0.41)$ \\
\hline All at one's own expense & \\
\hline All public expenses & \\
\hline Types of chronic diseases & \\
\hline 0 & \\
\hline 1 & \\
\hline
\end{tabular}


Table 2

Ability grades of the older people

\begin{tabular}{|lllll|}
\hline Ability grades & \multicolumn{3}{c|}{ long-term care facilities } & \multirow{2}{*}{ Total[n,(\%)] } \\
\cline { 2 - 4 } & 1[n,(\%)] & 2[n,(\%)] & 3[n,(\%)] \\
\hline Ability intact & $16(8.5)$ & $40(11.7)$ & $0(0.0)$ & $56(7.7)$ \\
\hline Mild disability & $81(42.9)$ & $204(59.6)$ & $11(5.7)$ & $296(40.8)$ \\
\hline Moderate disability & $23(12.1)$ & $36(10.5)$ & $19(9.8)$ & $78(10.6)$ \\
\hline Severe disability & $79(36.5)$ & $62(18.1)$ & $164(84.5)$ & $295(40.7)$ \\
\hline Total & $189(26.1)$ & $342(47.1)$ & $194(26.8)$ & $725(100.0)$ \\
\hline
\end{tabular}

\subsection{Item screening and initial scale formation}

\subsection{1 | Screening results of InterRAI-LTCF scale items}

Taking the nursing time of the elderly as dependent variables, 196 items of InterRAI-LTCF as independent variables, 154 items with statistical significance in the one-way ANOVA were used as independent variables, and the multiple regression analysis was performed by setting (a) in $=0.05$, (a) out $=0.10$. The results showed that 17 items could jointly explain $78.10 \%$ of the variance of nursing time (Table 3). 
Screening results of InterRAI-LTCF scale items $(n=725)$

\begin{tabular}{|c|c|c|c|c|c|c|}
\hline Dimension & Items & B & SD & Beta & $t$ & $P$ value \\
\hline & Constant & 149.948 & 143.953 & & 1.042 & 0.298 \\
\hline Communication and Vision & Listening & -98.597 & 43.114 & 0.047 & 2.287 & 0.023 \\
\hline Emotion and behavior & Always complaining and worrying & 186.790 & 62.619 & 0.057 & 2.983 & 0.003 \\
\hline Mental health & Telling or hinting being lonely & 251.279 & 100.946 & 0.048 & 2.489 & 0.013 \\
\hline \multirow[t]{5}{*}{ Functional condition } & Lower body dressing ability & 176.025 & 43.273 & 0.207 & 4.068 & 0.000 \\
\hline & Using of bathrooms & 184.065 & 35.033 & 0.253 & 5.254 & 0.000 \\
\hline & Bed activity & 84.892 & 40.627 & 0.094 & 2.090 & 0.037 \\
\hline & Eating & 167.764 & 38.839 & 0.167 & 4.319 & 0.000 \\
\hline & Time out of residence in the last three days & 106.799 & 38.624 & 0.063 & 2.765 & 0.006 \\
\hline Self-control & Controlled defecation & 126.243 & 36.476 & 0.118 & 3.461 & 0.001 \\
\hline \multirow[t]{2}{*}{ Disease diagnosis } & Hemiplegia & 389.993 & 121.416 & 0.060 & 3.212 & 0.001 \\
\hline & Pneumonia & 331.227 & 150.510 & 0.043 & 2.201 & 0.028 \\
\hline Skin condition & Foot problem & 94.233 & 47.939 & 0.039 & 1.966 & 0.050 \\
\hline \multirow[t]{3}{*}{ Treatment } & Respirator or ventilator & 860.899 & 282.062 & 0.059 & 3.052 & 0.002 \\
\hline & Wound care & 325.900 & 95.046 & 0.064 & 3.429 & 0.001 \\
\hline & Doctor's advice & 504.089 & 112.103 & 0.084 & 4.497 & 0.000 \\
\hline \multirow[t]{2}{*}{ Social participation } & Living ability & 277.054 & 52.950 & 0.210 & 5.232 & 0.000 \\
\hline & Time / space orientation & 84.635 & 40.670 & 0.067 & 2.081 & 0.038 \\
\hline \multicolumn{7}{|c|}{ Note: $R^{2}=0.781, \triangle R^{2}=0.776, p<0.001$} \\
\hline
\end{tabular}

\subsubsection{Screening results of AAQ scale items}

Taking the nursing time of the elderly as dependent variables, 40 items of AAQ as independent variables, and 32 items of statistical significance in single factor analysis of variance as independent variables. Also, 24-hour nursing time of the elderly was taken as dependent variable, and the multiple regression analysis was performed by setting (a) in $=0.05$, (a) out $=0.10$. The results showed that 9 items of the scale entered the regression equation $(F=131.210, P=0.000)$. These nine factors could explain $82.60 \%$ of the variation of nursing working hours (Table 4 ). 
Table 4

Screening results of $A A Q$ scale items $(n=725)$

\begin{tabular}{|c|c|c|c|c|c|c|}
\hline Dimension & Items & B & SD & Beta & $\mathbf{t}$ & $P$ value \\
\hline & Constant & 4436.539 & 231.340 & & 19.178 & 0.000 \\
\hline Disease diagnosis & Dementia & 154.512 & 44.595 & 0.080 & 3.465 & 0.001 \\
\hline \multirow[t]{6}{*}{ Activities of daily life } & Eating & 80.597 & 16.744 & 0.159 & 4.813 & 0.000 \\
\hline & Making up & 119.477 & 30.238 & 0.137 & 3.951 & 0.000 \\
\hline & Dressing & 78.106 & 21.409 & 0.162 & 3.648 & 0.000 \\
\hline & Stool control function & 41.768 & 16.153 & 0.080 & 2.586 & 0.010 \\
\hline & Bathroom usage & 88.547 & 24.809 & 0.178 & 3.569 & 0.000 \\
\hline & Indoor movement & 32.943 & 13.794 & 0.096 & 2.388 & 0.017 \\
\hline \multirow[t]{2}{*}{ Social participation } & Time / space orientation & 118.066 & 36.303 & 0.092 & 3.252 & 0.001 \\
\hline & Living ability & 223.464 & 46.010 & 0.156 & 4.857 & 0.000 \\
\hline
\end{tabular}

Note: $R^{2}=0.826, \triangle R^{2}=0.824, p<0.001$

Abbreviations: B, partial regression coefficient; SD, standard deviation; Beta, Standard regression coefficient; AAQ, Ability assessment questionnaire for older people.

\subsubsection{Initial scale formation}

The research team selected 8 nursing management experts in the field of elderly care and nursing working in the frontline for focus interviews. Combined with the objective screening method and focus interview method, the qualified parts of each scale are selected as scale items, and a scale was initially formed with a total of 6 dimensions, namely Mentation and cognitive, Perception and communication, Emotional problems, Mental and behavioral problems, Daily life and social participation, and Skin and oral status, a total of 42 items, including 24 items of InterRAI-LTCF scale and 18 items of AAQ.

\subsection{The validity}

\subsection{1 | Content validity}

In this study, seven experts related to elderly care service research in Nanjing were invited to judge the scale and items respectively. The recovery rate was $100 \%$, and positive coefficient of the expert was $100 \%$. The expert's judgment on the problem was based on (Ca) of 0.86 , familiarity of (Cs) of 0.83 , and authority coefficient of $(\mathrm{Cr})$ of 0.85 . These experts are highly authoritative in elderly care. There were options of a four-grade score set in the letter table, among which $1=$ irrelevant, $2=$ weak correlation, $3=$ strong correlation, 4 = very relevant. The percentage of S-CVI/UA (universal agreement) was $87.5 \% \varangle 80 \%$, which indicated that the content validity of the scale was good. The S-CVI/Ave value of the scale was $0.982>0.9$, which met the requirements.

\subsubsection{Construct validity: Confirmative factor analysis}

We tested the model with the six dimensions formed in the previous stage. The item factor loading results showed that the factor load of the two items, T27 "Time to get out of residence in the last three days" and T40 "The family maintains a strong relationship of mutual assistance", were less than 0.4 and negative for the first analysis, so they were deleted. After deleting these 
two items, The CFA model reached a good fit, with $\chi 2=6434.051, \mathrm{df} 725, \mathrm{p}<0.01 ; \mathrm{CFI}=0.970 ; \mathrm{TLI}=0.953 ; \mathrm{RMSEA}=0.043 ; \mathrm{SRMR}=$ 0.061. After the modification of the model, each index showed that the modified model fitted well with the actual data model. The secondary order factor was good for every dimension (Fig. 1).

\subsection{3 | The reliability}

The reliability coefficient alpha was 0.951 for the entire scale, and for each factor, that would be 0.906 for Mentation and cognitive, 0.849 for Perception and communication, 0.763 for Emotional problems, 0.768 for Mental and behavioral problems, 0.946 for Daily life and social participation, and 0.760 for Skin and oral condition respectively.

\subsection{Scale classification}

\subsection{1 | Establishment of the best $\mathrm{K}$ value}

Matlab2015b was used as a statistical tool, and the results of the comprehensive evaluation of 246 elderly people collected for the second time was used as a data set. K values were randomly assigned 2-10 to classify the data set and calculate the four validity indicators of different classification results (Table 5, Fig. 2).

Table 5

Results of evaluation indicators with different $k$ values $(n=246)$

\begin{tabular}{|lllllllllll|}
\hline Indicators & $\mathrm{K}=\mathbf{2}$ & $\mathrm{K}=\mathbf{3}$ & $\mathrm{K}=\mathbf{4}$ & $\mathrm{K}=\mathbf{5}$ & $\mathrm{K}=\mathbf{6}$ & $\mathrm{K}=\mathbf{7}$ & $\mathrm{K}=\mathbf{8}$ & $\mathrm{K}=\mathbf{9}$ & $\mathrm{K}=\mathbf{1 0}$ \\
\hline SSE & 991.1484 & 830.2628 & 561.5667 & 531.9026 & 498.3412 & 442.3305 & 396.5902 & 378.302 & 334.2205 \\
\hline SSB & 723.8516 & 884.7372 & 1153.433 & 1183.097 & 1216.659 & 1272.669 & 1318.410 & 1336.698 & 1380.779 \\
\hline IntraDPS & 0.577929 & 0.484118 & 0.327444 & 0.310147 & 0.290578 & 0.257919 & 0.231248 & 0.220584 & 0.194881 \\
\hline InterDPS & 0.422071 & 0.515882 & 0.672556 & 0.689853 & 0.709422 & 0.742081 & 0.768752 & 0.779416 & 0.805119 \\
\hline
\end{tabular}

Note: $p<0.001$

Abbreviations: SSE, within-cluster sum of squared errors; SSB, single side band ; IntraDPS, Intra-Cluster part of Dispersion; InterDPS, Inter-Cluster part of Dispersion.

\subsection{2 | Cluster analysis results}

Cluster analysis was conducted according to the scores of each dimension and the total score of CAOPAs, and the comprehensive ability of older people can be divided into four grades. As for the result of test, there were 21 older people in grade 0 (ability intact), 52 in grade 1 (Mild disability), 142 in grade 2 (moderate disability) and 31 in grade 3 (severe disability).

\subsection{3 | Discriminant analysis results}

According to the clustering results, discriminant analysis was carried out on the non-overlapping parts of the comprehensive ability assessment scores of older people, and the discriminant function was established (Table 6). The constructed discriminant function was used in this study to cross verify the non-overlapping data, among which the correct discrimination rates of four grades were $95.2 \%, 96.2 \%, 95.8 \%$ and $93.5 \%$, indicating that the established discriminant function had good discrimination effect (Table 7). 
Table 6

Discriminant function coefficient of comprehensive ability grade

\begin{tabular}{|lllll|}
\hline \multicolumn{5}{|c|}{ Discriminant function } \\
\hline & 1 & 2 & 3 & 4 \\
\hline Mentation and cognitive & 1.165 & 1.016 & 0.161 & 0.422 \\
\hline Perception and communication & 0.196 & 0.551 & -0.06 & 0.017 \\
\hline Emotional problems & 0.758 & -0.558 & 0.29 & 4.336 \\
\hline Mental and behavioral problems & 5.965 & 1.385 & -0.007 & -0.47 \\
\hline Daily life and social participation & 0.389 & 0.533 & 0.119 & 0.131 \\
\hline Skin and oral condition & 0.573 & 1.237 & 1.148 & 2.118 \\
\hline (Constant) & -31.197 & -21.487 & -2.739 & -12.364 \\
\hline Note: $\mathrm{p}<0.001$ & & & & \\
\hline
\end{tabular}

Table 7

Cross validation of comprehensive ability assessment of older people [n(\%)]

\begin{tabular}{|llllll|}
\hline Grades & \multicolumn{2}{l}{ Forecast group members } & \multicolumn{2}{l}{ Total } \\
\cline { 2 - 5 } & $\mathbf{1}$ & $\mathbf{2}$ & $\mathbf{3}$ & $\mathbf{4}$ & \\
\hline 1 & $20(95.2 \%)$ & $1(4.8 \%)$ & 0 & 0 & $21(100 \%)$ \\
\hline 2 & $2(3.8 \%)$ & $50(96.2 \%)$ & 0 & 0 & $52(100 \%)$ \\
\hline 3 & 0 & 0 & $136(95.8 \%)$ & $6(4.2 \%)$ & $142(100 \%)$ \\
\hline 4 & 0 & 0 & $2(6.5 \%)$ & $29(93.5 \%)$ & $31(100 \%)$ \\
\hline Note: $p<0.001$ & & & & \\
\hline
\end{tabular}

The discriminant function was used to discriminate and analyze the overlapping data, and finally established the segmentation boundary value of each grade (Table 8 ).

Table 8

Classification method of CAOPAs

\begin{tabular}{|ll|}
\hline Grades & Score range \\
\hline Grade 0 (ability intact) & $0-35$ points \\
\hline Grade 1 (Mild disability) & $36-51$ points \\
\hline Grade 2 (moderate disability) & $52-80$ points \\
\hline Grade 3 (severe disability) & $81-110$ points \\
\hline Note: $\mathrm{p}<0.001$ & \\
\hline Abbreviations: CAOPAs, Comprehensive Ability of Older People Assessment scale. \\
\hline
\end{tabular}

\section{5 | Reverse verification}


In order to verify the practicability and rationality of CAOPAs, the correlation coefficient was used to determine which scale was more convincing. A total of 246 elderly people were evaluated by two scales, and 63 of them had different ability grades.

Spearman correlation analysis was conducted to the ability grades $(1,2,3,4)$ of these 63 elderly people and the Ability related modules in InterRAI-LTCF. The results showed that the correlation coefficient of CAOPAs is higher than that of AAQ, indicating that CAOPAs is more related to the ability of the elderly, thus the evaluation results and grades are more convincing (Table 9).

Table 9

Spearman correlation analysis results $(n=63)$

\begin{tabular}{|lll|}
\hline & CAOPAs & AAQ \\
\hline CAOPAs & 1.000 & 0.138 \\
\hline AAQ & 0.138 & 1.000 \\
\hline Body mass index & $-0.263^{\star \star}$ & -0.124 \\
\hline Cognitive score & $0.535^{\star \star}$ & 0.018 \\
\hline Activities of daily living & $0.511^{\star \star}$ & 0.045 \\
\hline Depression score & -0.075 & 0.085 \\
\hline Pain score & $-0.340^{\star \star}$ & $-0.169 *$ \\
\hline Communication score & $0.453^{\star \star}$ & -0.071 \\
\hline Pressure ulcer score & $0.490^{\star \star}$ & 0.039 \\
\hline social participation & $-0.277^{\star *}$ & 0.025 \\
\hline $\begin{array}{l}\text { Abbreviations: CAOPAs, Comprehensive Ability of Older People Assessment scale; AAQ, Ability assessment questionnaire for } \\
\text { older people. }\end{array}$ & \\
\hline
\end{tabular}

\section{4 | Discussion}

We have developed a new instrument for assessing comprehensive ability in older people called CAOPAs and appraised its psychometric characteristics. The theoretical framework considered was the holistic nursing theory, which is guided by modern nursing concept, requiring the best nursing measures for older people's physiological, psychological, social participation, cultural adaptation and other aspects of nursing needs[16]. The instrument showed good validity and reliability. Therefore, it could be used by pension evaluators to assess the comprehensive ability of the elderly.

\subsection{The Scientific Development of CAOPAs}

\subsection{1 | This study used mature scales for evaluation, combined with nursing time measurement method for verification}

Under the framework of holistic nursing theory and with help of the previous literature research and empirical investigation of the research team, the scales, which have been published whether at home or abroad and have been tested for reliability and validity, were selected to conduct field surveys for pension institutions. InterRAI-LTCF and AAQ were used to assess the ability and nursing needs of the same elderly. Both scales are internationally recognized mature and reliable assessment tools. Each item of the maturity scale has a detailed description as the basis for evaluation and judgment, and they have been used in the elderly population, which conformed to our research purpose and direction. The pre-research results of the subject, "Nursing Time Measurement Table for Elderly Nursing Service Projects" and the information chemical time measurement platform were used to measure the nursing care time for the elderly. In this study, it is the first time that the nursing time measurement method was used to select the scale items to explore the relationship and correlation between the comprehensive ability of the elderly and the length of nursing time, in line with the actual situation of long-term care institutions.

4.1.2 I This study combined quantitative and qualitative methods, as well as reliability and validity testing methods, aiming at making the scale construction more reasonable 
We used the data collected from the field survey to initially screen the scale items, and then combined the results of focus interview method to test and select the applicability and feasibility of the scale items to form a preliminary table. Based on the combination of qualitative and quantitative methods, the content validity and reliability of CAOPAs were tested. In the content validity assessment, there were deleted items considered not relevant. Then we obtained a good content validity index, confirming that the remaining items adequately represented the construct. Confirmatory factor analysis was used to judge the structural validity of the scale, to verify the fitting degree of theoretical model and data and to revise the naming and items of factors after confirmatory factor analysis. The results showed that the construction of the scale was more scientific, reasonable and standardized.

\subsection{The rationality of the classification of CAOPAs}

In this study, through large sample experiment, cluster sampling method, cluster analysis, discriminant analysis and the establishment of the best $\mathrm{K}$ value were used to divide the scores of comprehensive ability assessment of the elderly by sections and to establish the boundary value of each segment. The best clustering number ( $k$ value) was 4 . Combined with the suggestions of elderly care service experts and the requirements of national policy documents, the comprehensive ability assessment of the elderly in nursing institutions is divided into four grades: Grade 0 (ability intact), Grade 1 (Mild disability), Grade 2 (moderate disability) and Grade 3 (severe disability). Therefore, the ability of 246 elderly people is divided into four grades, including 21 older people in Grade 0, 52 in Grade 1, 142 people in Grade 2 and 31 in Grade 3. According to the results of cluster analysis, discriminant analysis was carried out on the non-overlapping part of CAOPAs, and the discriminant function was established. By using the established discriminant function to the known overlapping sample data, the correct discrimination rate of the four grades were $95.2 \%, 96.2 \%, 95.8 \%$ and $93.5 \%$, which showed that the established discriminant function had good discrimination effect and the final threshold value of each segment of the grading level was reasonable and reliable.

\subsection{The Feasibility of application of CAOPAs}

The empirical study was carried out after the formation of the final version of CAOPAs, and the analysis of variance between different grades of elderly people and nursing time was carried out. The results were in line with the actual situation of the older people's nursing needs. It could be seen that there were differences in the length of nursing time for the elderly of different ability grades with discrimination. The correlation analysis between the comprehensive ability assessment score of the elderly and nursing time showed that $r=0.358, P<0.05$. The results showed that the longer the nursing time of the elderly, the higher the comprehensive ability evaluation score of the elderly, and the higher the ability level of the elderly, showing that the nursing workload of the elderly care institutions is related to the ability level of the elderly. It also shows that the scale compiled by this project is scientific and effective, and conforms to the actual situation of the elderly care institutions. Therefore, CAOPAs are feasible in long-term care institutions.

\subsection{LIMITATIONS}

There is some limitations in this study. Firstly, the traceability of the older people may have influenced the answers to the questionnaire. Secondly, the adjustment of the structural validity of the scale is a long-term process. In this study, two values of confirmatory factor analysis are close to the standard of good fitness, indicating that the scale needs to be revised and improved in practice. In addition, due to the limited time of the survey, only four long-term care facilities were selected in this study, thus the results may only be the representation of the local reality. We must consider future evaluations of the psychometric characteristics of CAOPAs to confirm our results. However, as the first study that is carried out in many long-term care institutions and taking the screening of scale items and nursing working time into account in the comprehensive ability assessment of the elderly, this study is still commendable.

\section{5 | Conclusion}

The CAOPAs (40-item and 4-grade) is the scale which can accurately and comprehensively assess the older people's ability and nursing needs, whose results indicate that CAOPAs have good validity and reliability, as well as good usability and utility. The research results provide a reference basis for the elderly's nursing needs assessment and may deepen the reform of pension

Page $13 / 18$ 
services. In addition, CAOPAs monitoring can be useful for evaluating the effect of different nursing measures or pension service support over time and improve quality in nursing care for older people.

\section{Abbreviations}

CGA: Comprehensive geriatric assessment:

InterRAI-LTCF: International Resident Assessment Instrument-Long-term care facilities

AAQ: The Ability assessment questionnaire for older people

MDS: Minimum data set

ADL: Activities of daily living

CAOPAs: The Comprehensive Ability of Older People Assessment scale

Item-level CVI, I-CVI : Content validity of the item

Scale-level CVI, S-CVI: Content validity index of scale grade

CFA: Confirmative factor analysis

CFI: Comparative fit index

RMSEA: The root mean square error of approximation

TLI: Tucker Lewis index

SRMR: Standardized root mean square residual

SSE: Sum of squared errors

SSB: Single side band

IntraDPS(K): Intra-Cluster part of Dispersion

InterDPS(K): Inter-Cluster part of Dispersion

\section{Declarations}

\section{Ethics Approval and Consent to Participate}

This study was approved by Nanjing University of Chinese Medicine Ethics Committee. Before data collection, signed informed consent were obtained from all participants. Based on the ethical principles of informed consent, respect, beneficence and confidentiality, as honoured by the research team, all participants were informed about the aims and the method of the study. In the case of the elderly with cognitive decline, the legal guardian or representative to provide consent to participate in our study. Benefits and possible risks associated with this study were also explained to the participants. Besides, all data were collected anonymously and archived in a password-protected computer. Only the primary investigator and research team members have access to the data.

\section{Consent to publish}

Not Applicable

Availability of Data and Materials

Page $14 / 18$ 
The datasets used and analysed during the current study available from the corresponding author on reasonable request.

\section{Competing Interests}

The authors declare no competing interests.

\section{Funding}

National Natural Science Foundation of China囚The Research of Grading Care Model For the Elderly Under the Guidings of the Theory of Health Management, Grant/Award Number: 71573140.

(The role the funder: $\mathrm{XGH}$ : Correspondent author, the role of the funding body in the design of the study and collection, analysis, and interpretation of data and in writing the manuscript is funding support)

\section{Authors' contributions}

Conceptualization: LWT.

Data curation: LWT,ZY,PYL.

Methodology:XGH.

Project administration: $\mathrm{XGH}$.

Software: LWT.

Writing - original draft: LWT,ZY,XGH.

Writing - review \& editing: LWT,PYL.

All authors read and approved the final manuscript.

\section{Acknowledgements}

Our sincere appreciation is extended to all the older people, elderly care service industry experts who participated in the study and the support and help of 4 long-term care facilities.

\section{References}

[1]World Health Organization. (2002). Active Aging: A Pllicy Framework. Geneva, Retrieved from http : // www. who. int/healthinfo/survey/ ageingdefnolder/en/

[2]Cardoso, R., Sá, S., Domingos, A. M., Sabóia, V. M., Maia, T. N., Padilha, J., \& Nogueira, G. A. (2018). Educational technology: a facilitating instrument for the elderly care. Revista brasileira de enfermagem, 71 Suppl 2, 786-792. https://doi.org/10.1590/00347167-2017-0129

[3]Álvarez-García, J., Durán-Sánchez, A., Del Río-Rama, M. C., \& García-Vélez, D. F. (2018). Active Ageing: Mapping of Scientific Coverage. International journal of environmental research and public health, 15(12), 2727.

https://doi.org/10.3390/ijerph15122727

[4]Pérez-Cuevas, R., Doubova, S. V., Bazaldúa-Merino, L. A., Reyes-Morales, H., Martínez, D., Karam, R., Gamez, C., \& MuñozHernández, O. (2015). A social health services model to promote active ageing in Mexico: design and evaluation of a pilot programme. Ageing and society, 35(7), 1457-1480. https://doi.org/10.1017/S0144686X14000361

[5]Walker A. (2015). Active ageing: realising its potential. Australasian journal on ageing, 34(1), 2-8. https://doi.org/10.1111/ajag.12219

Page $15 / 18$ 
[6]Ellis, G., \& Langhorne, P. (2005). Comprehensive geriatric assessment for older hospital patients. British medical bulletin, 71, 45-59. https://doi.org/10.1093/bmb/ldh033

[7]Paquay, L., De Lepeleire, J., Schoenmakers, B., Ylieff, M., Fontaine, O., \& Buntinx, F. (2007). Comparison of the diagnostic accuracy of the Cognitive Performance Scale (Minimum Data Set) and the Mini-Mental State Exam for the detection of cognitive impairment in nursing home residents. International journal of geriatric psychiatry, 22(4), 286-293.

https://doi.org/10.1002/gps.1671

[8]Morris, JN., Belleville-Taylor, P., Fries, BE et al., (2011) InterRAl long-term care facilities( LTCF) assessment form and user's

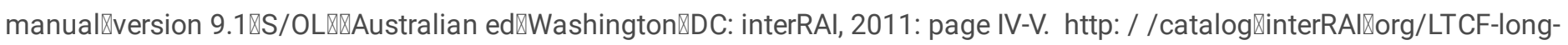
term-care-facilities-manual-australian

[9]Boorsma, M., Langedijk, E., Frijters, D. H., Nijpels, G., Elfring, T., \& van Hout, H. P. (2013). Implementation of geriatric assessment and decision support in residential care homes: facilitating and impeding factors during initial and maintenance phase. $B M C$ health services research, 13, 8. https://doi.org/10.1186/1472-6963-13-8

[10]China Social Welfare Association, Peking University School of Nursing, China Women Physicians Association, et al (2013). MZ/T039-2013 Older People's Ability Evaluation[S]. Beijing: China Standard Press, 2015: 1.

[11]Guo, H. Y., Wang, L., Wang, Z. W.,et al (2017). Research on the classification of the ability of the elderly [J]. Chinese Nursing Management, 2013, 13 (9): 35-38. DOI: 10.3969/j. issn.1672-1756.2013.09.012.

[12]Wang T. Y., Pan Z. X., He X. H., et al(2011). Analysis of related factors of sleep disorders in the elderly [J]. Journal of Contemporary Medicine, 2011, 18 (23): 74-76.

[13]Liu Yong, Electronic Reading Database of Colleges and Universities in Jiangxi Province.

[14]Nunnally, J. C., \& Bernstein, I. H. (1994). Psychometric theory. New York: McGraw-Hill.

[15]Shi, Q. C., (2017). A practical study on the classification of the ability of the elderly in pension institutions and the definition of care content [D]. Nanjing University of traditional Chinese Medicine.

[16]Chen, Y. Q., \& Luo, L. (2016). Effect of holistic nursing intervention on quality of life and clinical efficacy of hemodialysis patients with chronic renal failure [J]. Hebei Medicine, 2016,22 (8): 1370-1372.

\section{Figures}




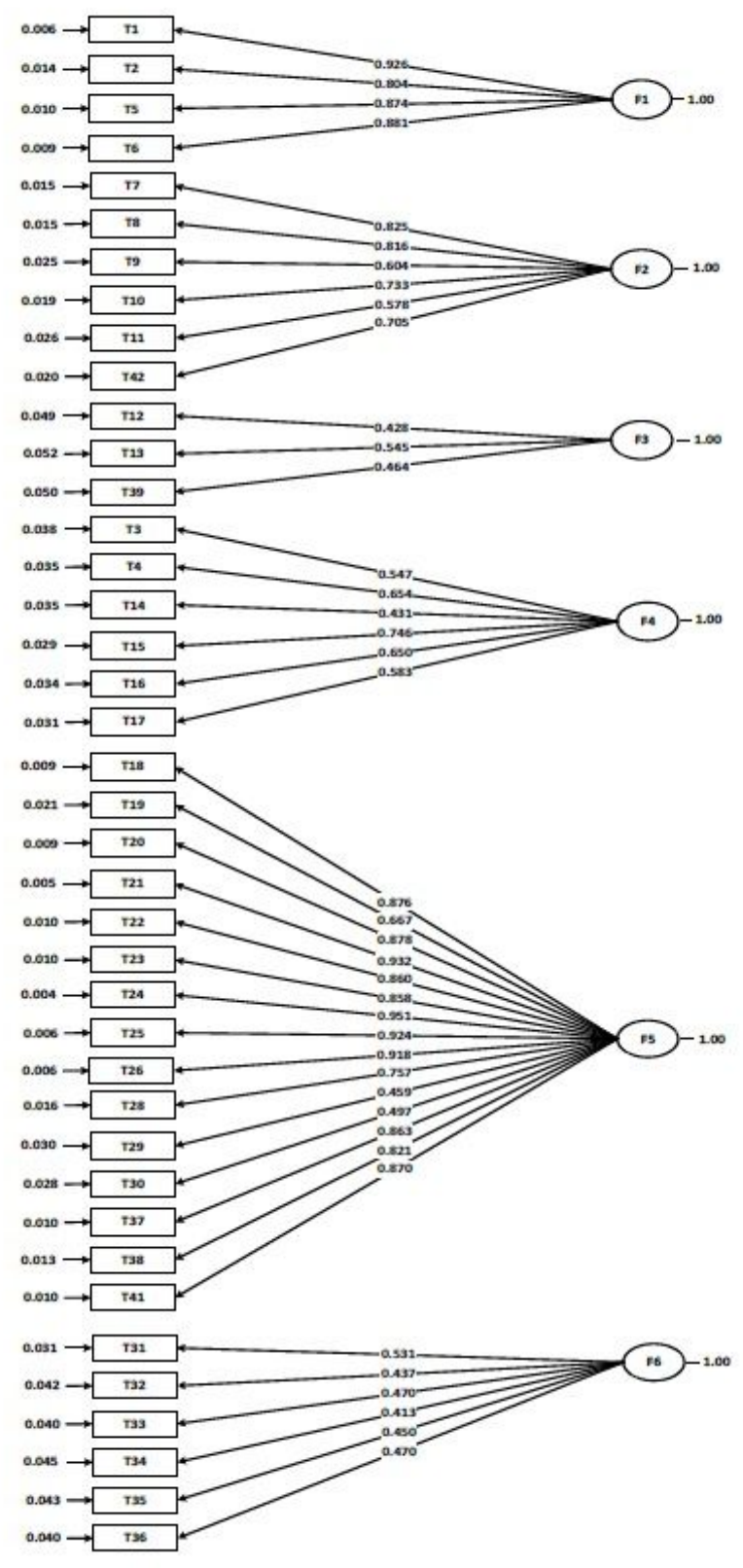

Figure 1

Confirmatory Factor Analysis model of CAOPAs. (CAOPAs, Comprehensive Ability of Older People Assessment scale; T, Item; F, Dimension).
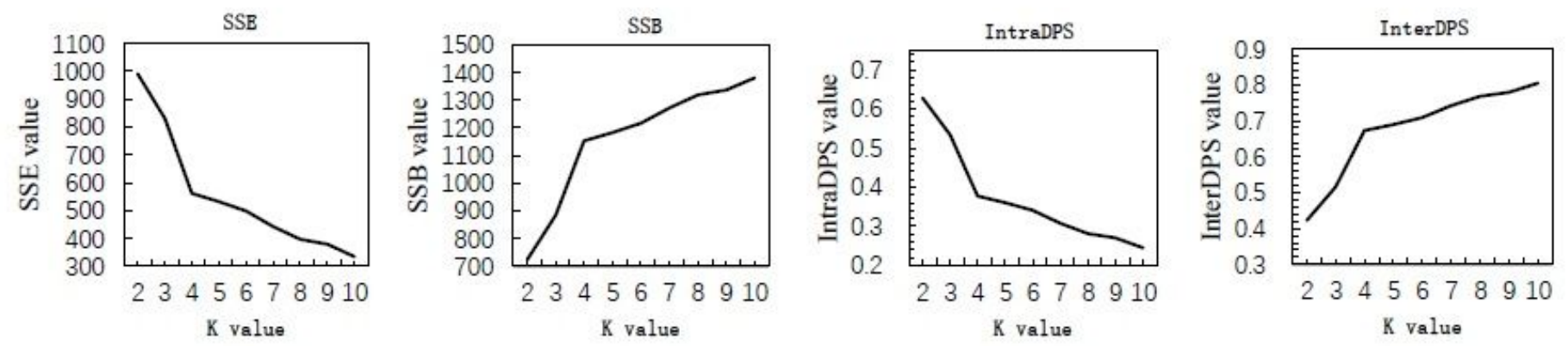

Figure 2 
4 indicator values corresponding to different $k$ values

Page 18/18 